\title{
VARIABILIDAD CLIMÁTICA EN EVENTOS EXTREMOS DE NIVEL DEL MAR Y OLEAJE
}

\author{
Melisa MENÉNDEZ GARCÍA \\ Instituto de Hidráulica Ambiental "IHCantabria" (Universidad de Cantabria). \\ menendezm@unican.es
}

\section{RESUMEN}

El análisis de los valores extremos asociados a temporales de oleaje y niveles del mar elevados juegan un papel relevante en los impactos marinos y costeros. Este trabajo presenta diferentes aspectos relativos al análisis estadístico de extremos para obtener información sobre la variabilidad climática en estas variables, tanto en su frecuencia de presentación como magnitud. Para ello, se han aplicado modelos estadísticos basados en la distribución generalizada de extremos (GEV) a partir de máximos mensuales permitiendo al modelo su variación en el tiempo. Los modelos se han aplicado sobre distintas fuentes de datos (mareógrafos, boyas, altimetría de satélite), teniendo en cuenta las peculiaridades de cada base de datos y comparando los resultados. Al modelar la variabilidad de los datos se analizan los ciclos estacionales en los periodos de retorno del oleaje y la influencia de patrones climáticos dominantes en las variaciones interanuales/decadales del nivel del mar. En particular la relevancia de la oscilación ártica (AO) sobre el hemisferio norte y la afección global del fenómeno del Niño.

Palabras clave: eventos extremos, temporales de oleaje, nivel del mar, variabilidad climática, modelos de extremos, no-estacionariedad.

\begin{abstract}
Extreme value analysis of sea surface dynamics (waves and sea level) play an important role in marine and coastal impacts. This paper presents different aspects of the statistical analysis of extremes to provide information on their climate variability, both in frequency and magnitude. This goal is achieved by applying time-dependent statistical models based on GEV (generalized extreme value) distribution from monthly maxima values. The models were applied on different data sources (tide-gauges, buoys, satellite altimetry), taking into account the peculiarities of each database and comparing the results. By modeling the climate variability, seasonal cycles of extreme waves and interannual variability of extreme sea levels are investigated through return value periods. In particular, the relevance of the Arctic Oscillation (AO) on the northern hemisphere and the overall influence of the El Niño phenomenon.
\end{abstract}

Key words: extreme events, wave storms, sea level, climate variability, extreme value models, time-dependence. 


\section{INTRODUCCIÓN}

Los extremos asociados al oleaje y nivel del mar juegan un papel relevante en los impactos marinos y costeros. Determinan la evolución geomorfológica costera y los ecosistemas asociados. Es por ello que la evaluación del riesgo de inundación, el diseño de obras marítimas o la gestión del territorio costero requieran de la caracterización de estos eventos extremos. En este contexto, los principales agentes de cambio de estas dinámicas marinas (ej. el aumento del nivel del mar, las tormentas) están controlados por la variabilidad del sistema climático a distintas escalas temporales, y la estimación de eventos asociados altos periodos de retorno (20 años, 100 años) puede estar condicionada por estas variaciones climáticas.

Este trabajo presenta diferentes aspectos relativos al análisis estadístico para tener en cuenta la no-estacionariedad en estas variables, tanto en su frecuencia de presentación como magnitud. Se han aplicado modelos matemáticos paramétricos dependientes del tiempos a partir de una selección de máximos en bloques temporales. Al modelar la variabilidad de los datos se analizan los ciclos estacionales en los periodos de retorno y la influencia de patrones climáticos en las variaciones interanuales/decadales.

El análisis de extremos sobre las variables nivel del mar y oleaje requiere el conocimiento del registro de datos del que se parte. Otro aspecto a tener en cuenta es que la mayoría de las teorías estadísticas parten de la premisa de trabajar con muestras aleatorias de observaciones independientes. En muchos casos el análisis de una serie temporal no suele cumplir esta premisa y observaciones sucesivas son dependientes entre si, por lo que es muy importante el orden temporal entre los valores de trabajo. Cuando los valores sucesivos son dependientes, como es el caso de la componente del nivel del mar debida a la marea astronómica, el análisis de la serie temporal permite predecir qué valores se van a alcanzar en el futuro a partir de observaciones en el pasado (serie determinista). Las series temporales de las variables nivel del mar y oleaje presentan la característica de que, conocidos los valores pasados de la serie, no es posible predecir con total certeza un valor futuro, es decir; son series no deterministas o aleatorias. Evidentemente aunque no sea posible predecir con total exactitud la serie temporal (o sus eventos extremos), para que tenga interés su estudio el resultado no puede ser completamente aleatorio. Existen algunas regularidades y patrones en cuanto a su comportamiento que deben estudiarse en el contexto de su marco climático, lo que hará posible su modelado. Si se encuentran patrones que caractericen los valores extremos podremos también describirlas mediante modelos basados en distribuciones de probabilidad. Así, los modelos de extremos se definen mediante el supuesto de que estamos trabajando con procesos estocásticos; es decir, la secuencia ordenada de variables aleatorias y su distribución de probabilidad asociada.

\section{MÉTODOS}

\subsection{Aspectos a considerar a partir de los datos de partida.}

Las fuentes de procedencia de las bases de datos disponibles de nivel del mar y oleaje se suelen clasificar cómo (i) observaciones (datos medidos a partir de instrumentación) y (ii) reconstrucciones históricas (hindcasts y reanalysis). A su vez, las observaciones pueden proceder de medidas in-situ (boyas para el oleaje y mareógrafos en el caso del nivel del mar) o medidas remotas (satélites). Las bases de datos procedentes de satélite con información sobre el oleaje y nivel del mar proceden de altimetría. 
Los datos procedentes de reconstrucciones históricas son simulaciones generadas mediante modelado numérico. Deben por tanto llevar asociados procedimientos de validación/corrección de los valores máximos frente a medidas observadas. Por otro lado, una vez verificada su calidad, presentan información continua y equi-espaciada en el tiempo, generalmente de suficiente duración para capturar la variabilidad climática (al menos 30 años). Además, permiten el desarrollo de estudios espaciales, en función de la resolución espacial utilizada durante el modelado.

Los registros medidos in-situ suelen tener una resolución horaria regular. Por otro lado, solo proporcionan información climática sobre extremos en una ubicación específica. Su principal inconveniente es la presencia de 'huecos' en la serie, especialmente durante algunos periodos asociados a los eventos extremos de mayor intensidad por inoperatividad de los sensores en campo. Mareógrafos y boyas presentan diferentes características para el análisis extremal. Existen numerosos mareógrafos con registros históricos largos, lo que facilita su caracterización del comportamiento de los eventos extremos. No obstante, los registros procedentes de mareógrafos requieren un análisis exhaustivo de calidad de datos, ya que sus series temporales suelen presentar discontinuidades, cambios de tecnologías en sus sensores y huecos de varios años, entre otros. Los registros de oleaje procedentes de boyas son más fiables en cuanto a su calidad, pero sus registros cubren periodos de tiempo mucho más cortos.

Las bases de datos procedentes de altimetría de satélite más utilizadas son generalmente conjuntos de datos multi-misión inter-calibrados. Hoy en día se dispone de más de 20 años de medidas, lo que permite obtener información sobre extremos en regiones costeras del mundo dónde no hay ninguna otra observación medida. Los datos procedentes de satélite tienen, por otro lado, el principal inconveniente de ser irregulares tanto espacialmente como temporalmente. Este hecho implica que en muchos casos los datos procedentes de satélite no miden el máximo del evento extremo. Es por ello, que su uso en el análisis de extremos es inusual y, en los pocos estudios que se ha llevado a cabo la aproximación más común consiste en combinar la información en áreas geográficas delimitadas con características climáticas similares (e.g. Alves and Young, 2003).

\subsection{Implementación de la variabilidad climática en los modelos de extremos}

El estudio de la influencia del cambio climático sobre los temporales de oleaje y valores extremos del nivel del mar es una de las cuestiones científicas que se están investigando en la actualidad. Para resolver esta cuestión es necesario entender la variabilidad estacional, interannual, decadal y de largo plazo sobre los eventos extremos, ya que podrían tener un comportamiento diferente a las climatologías medias.

La aproximación estadística que se plantea en este trabajo consiste en la implementación de la no-estacionariedad en los modelos estadísticos de extremos. Es decir, se asume que la probabilidad de que ocurra un evento extremo de oleaje y nivel del mar puede ser diferente en función de la estación del año, de la situación anual del patrón de circulación atmosférica asociado, o de cambios de largo plazo asociados al calentamiento global. En la figura 1a se representa la función de densidad de probabilidad de extremos estacionaria y un esquema de cómo resulta al tener en cuenta la dependencia temporal estacional (b), un tendencia de largo plazo (c) y una serie de modulaciones entre años (d). A continuación se describe la implementación de la 
dependencia temporal en el modelo de extremos GEV (Generalized Extreme Value), basado en el Teorema de Fisher-Tippet (Galambos, 1987).

a)

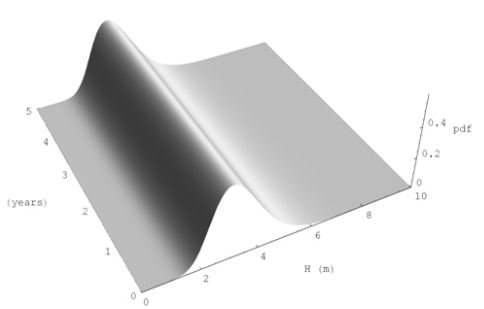

c)

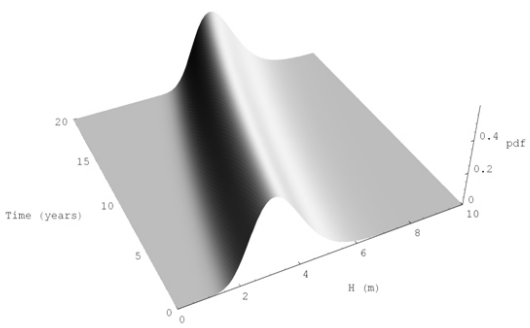

b)

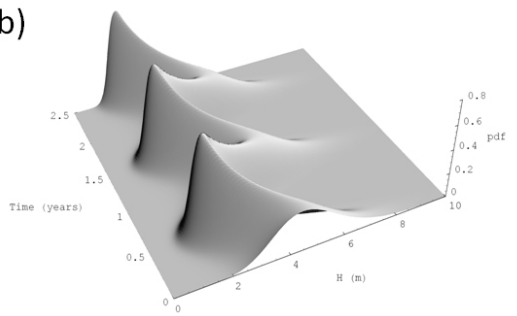

d)

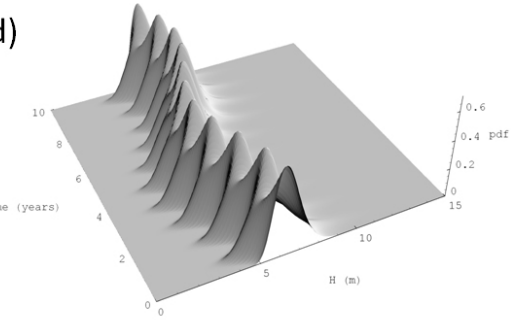

Fig. 1: Esquema de la función de densidad de probabildad estacionaria (a); dependiente del tiempo a escala estacional (b); incluyendo una tendencia de largo plazo (c), y variaciones estaciones e interanuales $(d)$.

La función de distribución GEV puede describirse mediante la expresión:

$G(x)= \begin{cases}\exp \left\{-\left[1+\xi\left(\frac{x-\mu}{\psi}\right)\right]_{+}^{-1 / \xi}\right\} & \xi \neq 0 \\ \exp \left\{-\exp \left[-\left(\frac{x-\mu}{\psi}\right)\right]\right\} & \xi=0,\end{cases}$

donde $[\mathrm{a}]_{+}$implica el $\max (\mathrm{a}, 0),-\infty<\mu<\infty$ es el parámetro de localización, $\psi>0$ es el parámetro de escala y $\xi$ es el parámetro de forma.

Si se considera que los parámetros de la distribución de probabilidad son funciones del tiempo: $\mu(t), \psi(t)$ y $\xi(t)$. El ajuste de modelo mediante máxima verosimilitud resulta en la siguiente expresión:

$$
\begin{aligned}
\ell\left(x_{M A X} ; \theta \mid t_{i}\right)= & -\sum_{i=1}^{N}\left\{\log \psi\left(t_{i}\right)+\left(1+\frac{1}{\xi\left(t_{i}\right)}\right) \log \left[1+\xi\left(t_{i}\right)\left(\frac{x_{i}-\mu\left(t_{i}\right)}{\psi\left(t_{i}\right)}\right)\right]\right. \\
& +\left[1+\xi\left(t_{i}\right)\left(\frac{x_{i}-\mu\left(t_{i}\right)}{\psi\left(t_{i}\right)}\right)_{+}^{-1 / \xi\left(t_{i}\right)}\right\}
\end{aligned}
$$




\section{APLICACIONES}

En este apartado se muestran algunos resultados obtenidos al aplicar los modelos de extremos no estacionarios siguiendo la aproximación descrita sobre distintas bases de datos con información sobre el oleaje y nivel del mar.

\subsection{Análisis de la estacionalidad de los temporales de oleaje.}

En primer lugar se muestra el comportamiento de los cuantiles asociados al periodo de retorno de 20 años para cinco ubicaciones a lo largo del litoral español (figura 4). Las bases de datos utilizadas son los registros desde 1983 hasta 2003 de las boyas RedExt de Puertos del Estado. El modelo de extremos utilizado en este caso parte de la selección de máximos mensuales y posterior análisis estadístico mediante modelado de ciclos anuales y semianuales en los parámetros de localización, escala y forma de la GEV (más detalles del modelo en Menéndez et al., 2009). Los resultados obtenidos para Bilbao y Gijón presentan un ciclo anual para el parámetro de localización y escala y el valor negativo del parámetro de forma, claramente situado en la estación de invierno (de noviembre a marzo). En el caso de La Coruña, el ciclo semianual en el parámetro de escala también es significativo. La localización occidental de la boya de La Coruña causa una estación de invierno más amplia que la observada en las boyas de Gijón y Bilbao, ya que está más expuesta a las borrascas que provienen del oeste. El conjunto de datos de Cádiz es el único que presenta un ciclo anual para el parámetro de forma; con una cola de la distribución corta en la estación de verano, debido a las tormentas locales, y una cola larga en la estación de invierno (noviembre a febrero), debido a los temporales marítimos generados en un área grande. La modulación de la estacionalidad mostrada en la boya de Valencia es un patrón muy conocido a lo largo de la costa mediterránea española. Presenta dos picos de máximos de altura de ola significante, uno resultante del fenómeno 'gota fría' en la estación de otoño (calentamiento del mar Mediterráneo junto con ciclones) y otro en la estación de primavera.

En segundo lugar, se presenta un análisis similar pero con cobertura espacial global a partir de la información del oleaje procedente de satélite. La base de datos utilizada cubre el periodo 1992-2010. Las medidas proceden de las misiones TOPEX, Jason1, Jason2, Envisat, GFO y ERS2 (http://www.aviso.oceanobs.com) y han sido calibradas mediante el método descrito en Cotton, 1998. Para lograr una buena representación espacial con suficiente datos de las trazas de los satélites, los datos se agregan en áreas geográficas dimensionadas por $3^{\circ}$ en longitud y $2^{\circ}$ en latitud. Posteriormente, en cada área se seleccionan los máximos mensuales testeando (i) independiencia entre máximos de máximos consecutivos (Méndez et al. 2007) y muestras con al menos el 20\% del máximo número de datos por área. A partir del modelo GEV dependiente del tiempo se ha permitido la variabilidad del ciclo estacional mediante funciones armónicas (más detalles en Izaguirre et al., 2011). Con los parámetros del modelo ajustados para cada celda se ha calculado el valor asociado al periodo de retorno de 20 años durante el invierno (diciembre, enero y febrero) y verano boreal (junio, julio y agosto). Los valores mayores durante el invierno boreal se localizan en el Atlántico y Pacífico Norte, mientras que los mayores extremos de oleaje durante el invierno austral (figura 5b) se localizan en el Océano Sur, en especial entre Sudáfrica y Australia, debido principalmente al fuerte régimen de vientos en esa región. Otro 
aspecto a destacar es la mayor variación estacional en los extremos en el hemisferio norte con respecto al hemisferio sur.
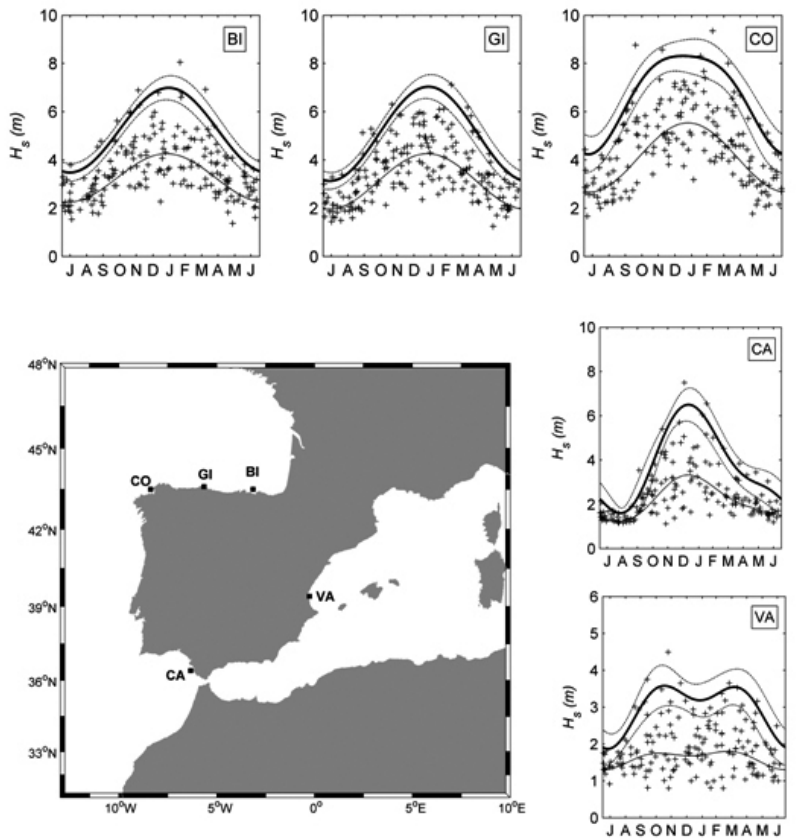

Fig 2. Resultados del modelado del régimen extremal estacional para las 5 boyas cuya ubicación se muestra en el mapa. Los periodos de retorno de 20 años se muestran en línea gruesa, con sus bandas de confianza al 95\%. La cruces representan los máximos mensuales medidos.

a)

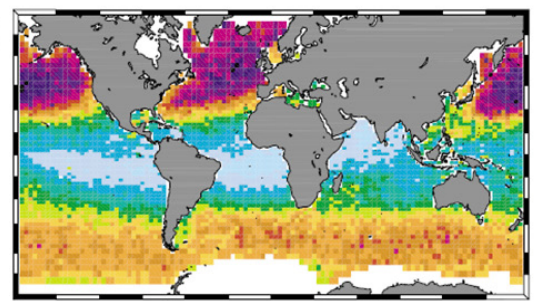

b)

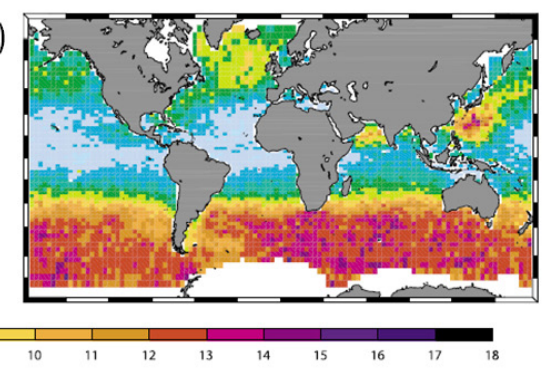

Fig 3. Valor extremo de altura de ola significante asociado a 20 años de periodo de retorno durante el invierno boreal ( $a, D E F)$ y verano (JJA, b). Figura modificada a partir de Izaguirre et al. 2011.

\subsection{Análisis de la variación interannual de los extremos del nivel de mar.}

A continuación se discuten y comparan los resultados más interesantes encontrados en los últimos trabajos realizados sobre el nivel del mar aplicando el modelo GEV no estacionario (más detalles del método en Menéndez et al. 2009). 
Es interesante destacar, por un lado la influencia de los patrones de circulación del sistema climático conocidos sobre los valores extremos observados del nivel del mar, que se introduce en el modelo de extremos como una co-variable que varía con el tiempo en el parámetro localización de la GEV. En particular, cabe destacar la significante influencia de los patrones teleconectivos AO (oscilación ártica, Thompson y Wallace, 1998) y el índice climático El Niño 3.4, descriptor del fenómeno del Niño.

La oscilación ártica es el patrón dominante de las variaciones interanuales sobre latitudes al norte de $20^{\circ} \mathrm{N}$. Se caracteriza por el gradiente de anomalías de la presión atmosférica entre el Ártico y la región localizada sobre las latitudes entre $37-45^{\circ} \mathrm{N}$. Está muy relacionada con el patrón de la Oscilación del Atlántico Norte (NAO). No obstante, su significancia en el modelo GEV para los extremos del nivel del mar se encuentra mejor definido para el índice AO que NAO. En la figura 4a se muestran los resultados obtenidos aplicando el modelo de extremos a partir de los datos de altimetría de las diferentes misiones Topex, Jason y OSTM (más información en PODAAC, podaac.jpl.nasa.gov) durante el periodo 1992-2012. En la figura 4b se muestran los resultados a partir de registros históricos sobre Europa de mareógrafos. Se puede apreciar cómo, a pesar de la diferencia en los datos de partida, los patrones espaciales son similares, son una relación positiva superior a $25 \mathrm{~cm}$ del nivel del mar en la región norte de Europa.

a)

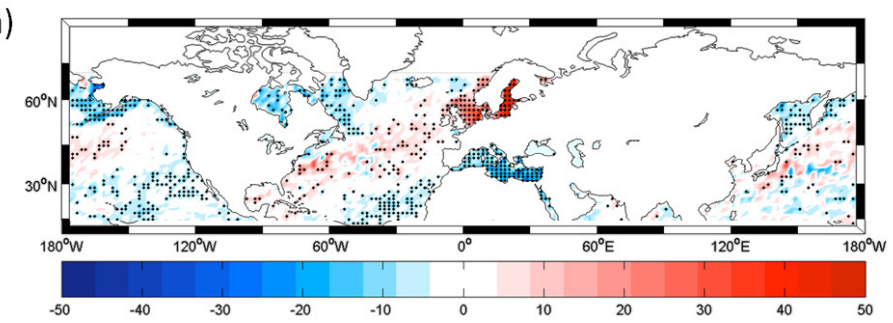

b)

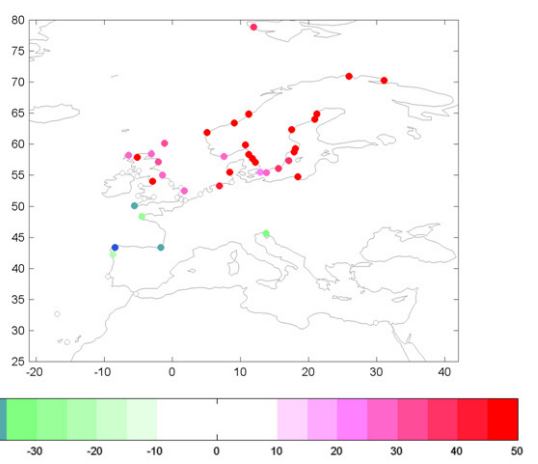

Fig 4. Sensibilidad del indice AO sobre los extremos del nivel del mar (cm/unidad de indice climático) a partir de información procedente de satélites (a) y de mareógrafos (b).

La influencia del fenómeno del Niño sobre el nivel del mar es ampliamente reconocida, no obstante el estudio de la distribución espacial de esta influencia sobre los 
extremos del nivel del mar requiere de más estudios. En la figura 5a se muestran los resultados obtenidos en Menéndez y Woodworth (2010) a partir de un análisis sobre un conjunto de datos de registros de mareógrafos mundial. En la figura $5 \mathrm{~b}$ se muestra el mismo resultado a partir de los datos de satélite procedentes de la NASA descritos anteriormente (agregados en áreas geográficas de $2^{\circ}$ de longitud por $1.5^{\circ}$ en latitud (Woodworth y Menéndez, 2015). Como se observa, los resultados obtenidos a partir de datos satelitales no solo concuerdan con las medidas de campo sino que permiten extender la información sobre la variabilidad interanual de los extremos del nivel del mar a regiones sin mediciones disponibles.

Los resultados verifican la asociación positiva entre el fenómeno del Niño y el nivel del mar extremal en cuanto a su variabilidad interanual en toda la costa oeste de América y el Pacífico tropical occidental. La propagación de la señal del Niño en el Océano Índico y en las costas nor-oeste de Australia. La relación positiva a lo largo de la costa oeste de los Estados Unidos indica una mayor probabilidad de un elevado nivel de agua durante los eventos Niño intensos. Por el contrario, el Pacífico tropical occidental y la costa de Australia muestran una sensibilidad con el signo inverso (ante eventos intensos de la Niña).

a)

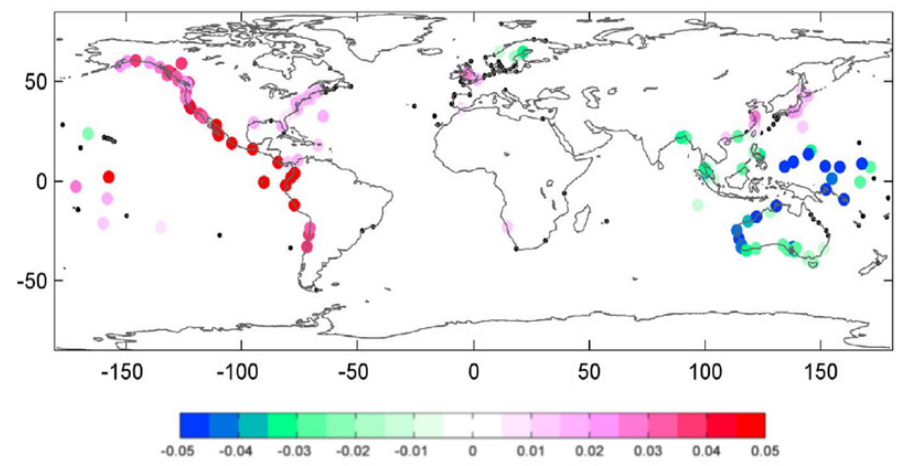

b)

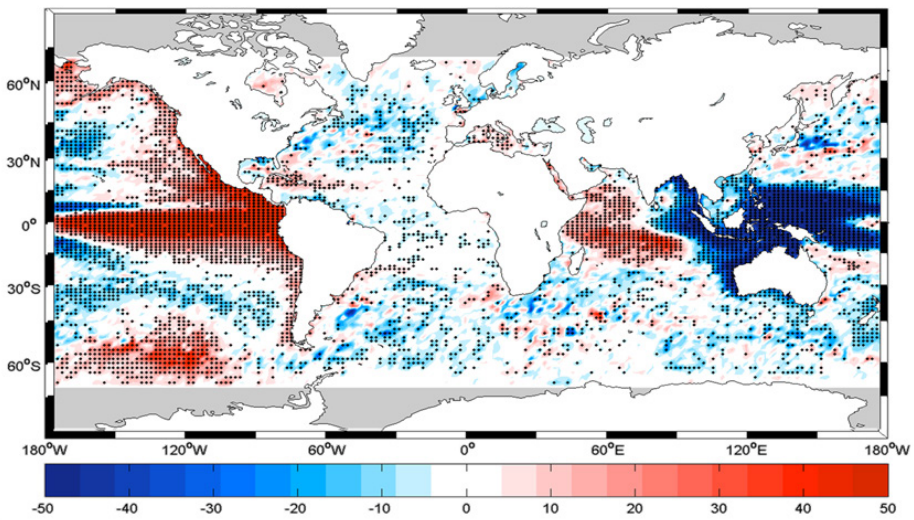

Fig 5. Sensibilidad del indice Niño3.4 sobre los extremos del nivel del mar (cm/unidad de indice climático) a partir de información procedente de mareógrafos (a) y de satélites (b). 


\section{REFERENCIAS}

Alves, J. H. G., \& Young, I. R. (2003). On estimating extreme wave heights using combined Geosat, Topex/Poseidon and ERS-1 altimeter data. Applied Ocean Research, 25(4), 167-186.

Cotton, P. D. (1998). A feasibility study for a global satellite buoy intercalibration experiment, Resolut. Consult. Rep. 26, Southampton Oceanogr. Cent., Southampton, U. K.

Galambos, J. (1987). The Asymptotic Theory of Extreme Order Statistics, Robert E. Krieger. Malabar, Florida. 2nd ed.

Izaguirre, C., Méndez, F. J., Menéndez, M., \& Losada, I. J. (2011). Global extreme wave height variability based on satellite data. Geophysical Research Letters, $38(10)$.

Menéndez, M., F. J. Méndez, and I. J. Losada (2009). Forecasting seasonal to interannual variability in extreme sea levels, ICES J. Mar. Sci., 66(7), 1490-1496, doi:10.1093/icesjms/fsp095.

Menéndez, M., F. J. Méndez, C. Izaguirre, A. Luceño, and I. J. Losada (2009). The influence of seasonality on estimating return values of significant wave height, Coastal Eng., 56(3), 211-219.

Menéndez, M., P. L. Woodworth (2010). Changes in extreme high water levels based on a quasi-global tide-gauge dataset, J. Geophys. Res., 115, C10011, doi:10.1029/2009JC005997.

Thompson, D. W. J., and J. M. Wallace (1998). The Arctic Oscillation signature in the wintertime geopotential height and temperature fields. Geophys. Res. Lett., 25, No. 9, 1297-1300.

Woodworth, P. L., Menéndez, M. (2015). Changes in the mesoscale variability and in extreme sea levels over two decades as observed by satellite altimetry. Journal of Geophysical Research: Oceans, 120(1), 64-77. 